\title{
高频重复经颅磁刺激联合高压氧治疗脑卒中后抑郁效果观察
}

Effect of High Frequency Repetitive Transcranial Magnetic Stimulation Combined with Hyperbaric Oxygen on Post-stroke Depression

李莉

$\mathrm{Li} \mathrm{Li}$

山东省济南市章丘区中医医院

中国・山东 章丘 250200

Zhangqiu District Hospital of Traditional Chinese Medicine, Jinan City, Shandong Province,

Zhangqiu, Shandong, 250200, China
【摘要】脑卒中疾病在脑血管疾病属于发病率较高的, 其常常伴有抑郁心理。论文解析了 高频重复经领磁刺激联合高压氧治疗脑卒中后抑郁的临床效果。

【Abstract】Stroke is a high incidence of cerebrovascular disease, often accompanied by depression. This paper analyzes the clinical effect of high frequency repetitive transcranial magnetic stimulation combined with hyperbaric oxygen in the treatment of post-stroke depression.

【关键词】高频重复经㘧磁刺激; 高压氧; 脑卒中; 抑郁; 临床数据

【Keywords】high frequency repetitive transcranial magnetic stimulation; hyperbaric oxygen; stroke; depression; clinical data

【DOI】10.36012/pmr.v1i1.158

\section{1 具体方法与过程分析}

方法: 遴选时间在 2018 年 6 月至 2019 年 6 月,合计确诊 脑卒中患者 165 例展开观察, 依据入院病例号为分组根据, 对 照组中有 82 例患者, 采用常规治疗手段; 治疗组中有 83 例患 者, 采用高频重复经颖磁刺激手段治疗, 收集 2 组的临床数 据, 以便于日后的对比分析。结果:治疗之前与治疗后 30 天 2 组患者的 HAMD 评分比较没有任何差异性, $P>0.05$ 无统计 学意义。而在患者治疗后当天, 治疗组中的 HAMD 评分却显 著低于对比组的 HAMD 评分, 组间数据对比有临床比较的意 义 $(P>0.05)$ 。展开临床治疗后对比组中轻度抑有症患者 8 例、 中度抑郁症患者 5 例、重度抑有症患者为 3 例, 抑郁率 $19.51 \%(16 / 82)$; 治疗组中轻度抑有症患者 1 例、中度抑有症 患者 1 例, 抑有率 $2.41 \%(2 / 83)$ 。对比组抑郁率比治疗组高, $P<$ 0.05 有比较性。结论: 对脑卒中抑有患者的治疗而言, 采用高 频重复经颅磁刺激联合高压氧的治疗效果更加显著, 且其具
有较高的安全性, 可展开临床推广与应用。

如今在生活水准不断提升的背景下, 人们的物质生活更 加的丰富, 随之各种疾病也会出现, 其中危害较大的便是脑血 管疾病, 有代表性的就有脑卒中, 其发病人群多是老年人。当 处于发病期间的时候, 患者会出现恐惧、焦虑抑郁的情况发 生, 让患者的正常治疗受到影响, 同时也对患者的生存质量产 生不良影响[1,2]。论文则是摘取 2018 年 6 月至 2019 年 6 月,合 计确诊脑卒中患者 165 例展开观察, 分析其应用高频重复经 颅磁刺激联合高压氧治疗后, 患者的抑有情况有怎样的改变。

\section{2 资料和方法}

\section{1 一般资料}

遴选时间在 2018 年 6 月至 2019 年 6 月, 合计确诊脑卒 中患者 165 例展开观察, 所有患者与国际疾病分类抑郁发作 诊断标准相符合, 且汉密尔顿抑郁量表(HAMD)的 24 项评分 均超过 20 分, 影响学检查 (CT) 也进一步确诊为脑卒中疾病。 
另外，需将有癫㾁病史的患者排余，将有精神病史的患者排 除, 将体内有金属植入的患者排余。依据入院病例号为分组根 据, 对比组中有 82 例患者, 入组男性 40 例, 入组女性 42 例, 最低年龄 52 岁,最大年龄为 83 岁, 中间值 $(62.1 \pm 4.2)$ 岁; 治疗 组中有 83 例患者, 入组男性 50 例, 入组女性 33 例, 最低年龄 50 岁, 最大年龄为 89 岁, 中间值 $(65.7 \pm 3.8)$ 岁。不论是性别, 还是年龄, 2 组患者均没有明显的差异性, 即 $P>0.05$ 具有统 计性。

\section{2 方法}

对比组给予患者基础抗抑郁治疗，即服用舍曲林、西酞普 兰等抗抑有的药物, 同时辅助音乐疗法以及中医针众疗法等, 以帮助患者缓解抑郁的心理。

治疗组给予患者基础抗抑有治疗+高频重复经颅磁刺激+ 高压氧治疗, 其中基础抗抑有治疗与对比组相同; 高频重复经 颅磁刺激治疗依据的仪器为：武汉依瑞德公司产 CCY-I 型磁 场刺激仪,其组成为圆形线圈, 主要是对患者的左侧背外侧前 额叶区展开刺激, 同时使得线圈和头皮呈现为切线, 相应的刺 激参数需要根据患者情况展开调整, 通常其频率为 $10 \sim 15 \mathrm{~Hz}$, 强度设置在 $90 \%$ 运动阈值, 每隔 $10 \mathrm{~s}$ 刺激一次, 一次时间是 $1 \mathrm{~s}$, 总的脉冲串为 1200 次 $/ \mathrm{d}$, 治疗周期是 10 天; 高压氧治疗, 当患者的病情较为稳定之后, 其将压力控制在 $0.2 \sim 0.3 \mathrm{Mpa}$, 加 压 $15 \mathrm{~min}$ 左右展开治疗, 当高压氧压力维持稳定后, 患者需要 吸入纯氧治疗 $0.5 \mathrm{~h}$, 治疗间歇为 $10 \mathrm{~min}$, 随后展开 2 次高压氧 吸入治疗, 共治疗 $0.5 \mathrm{~h}$, 当治疗完成之后需进行减压工作, 时 间则控制在 $25 \mathrm{~min}$ 左右, 一天治疗一次, 10 天为一个周期, 合 计治疗 3 5 个周期。

\section{3 统计学处理}

录入软件是 SPSS28.0 统计软件, 将文中患者的抑有数据 实施分析, 各计量数据选择 $\bar{x} \pm \mathrm{s}$ 表示, 组间数据以 $t$ 检验, 计数 资料选择 $\mathrm{n} / \%$ 表示, 以 $\chi^{2}$ 检验, $P<0.05$ 代表各数据间对比存在 统计学意义。

\section{3 结果}

\section{1 对 2 组患者治疗前后的 HAMD 评分比较}

治疗之前与治疗后 30 天 2 组患者的 HAMD 评分比较没 有任何差异性, $P>0.05$ 无意义(表 1 )。而在患者治疗后当天, 治疗组中的 HAMD 评分却显著低于对比组的 HAMD 评分, 组 间数据对比有临床比较的意义 $(P>0.05)$ 。

\subsection{2 组患者治疗后抑郁情况说明}

展开临床治疗后对比组中轻度抑有症患者 8 例、中度抑 郁症患者 5 例、重度抑有症患者为 3 例，抑郁率 19.51\% (16/
表 1 对 2 组患者治疗前后的 HAMD 评分比较 $[n /$ 分 $/ \bar{x} \pm s]$

\begin{tabular}{c|c|c|c|c}
\hline 组别 & 例数 & 治疗前 & 治疗后当天 & 治疗后 30 天 \\
\hline 治疗组 & 83 & $31.28 \pm 7.81$ & $16.92 \pm 5.01$ & $15.81 \pm 4.38$ \\
\hline 对比组 & 82 & $32.61 \pm 5.62$ & $22.37 \pm 3.73$ & $15.95 \pm 4.27$ \\
\hline$t$ & & 0.9649 & 5.8426 & 0.8135 \\
\hline$P$ & & 1.8352 & 0.0067 & 2.4874 \\
\hline
\end{tabular}

注: $t$ 为 2 组数据相似度大小的参数, $P$ 为 $\chi^{2}$ 检验中假设为真时的 概率。

表 22 组患者护理干预后抑郁情况对比分析 $(n / \%)$

\begin{tabular}{c|c|c|c|c|c}
\hline 组别 & 例数 & 轻度 & 中度 & 重度 & 抑有率 \\
\hline 治疗组 & 83 & 1 & 1 & 0 & $2(2.41)$ \\
\hline 对比组 & 83 & 8 & 5 & 3 & $16(19.51)$ \\
\hline$\chi^{2}$ & & & & & 14.0545 \\
\hline$P$ & & & & & 0.0001 \\
\hline
\end{tabular}

注: $\chi^{2}$ 为实际观测值与理论推断值之间的偏离程度, $P$ 为假设为 真时的概率。

82 ); 治疗组中轻度抑有症患者 1 例、中度抑有症患者 1 例, 抑 郁率 $2.41 \%(2 / 83)$ 。对比组抑有率比治疗组高, $P<0.05$ 有比较 性。

\section{3 患者神经缺损功能比较}

治疗之前, 治疗组与对比组的平均神经功能缺损评分是 $(25.17 \pm 4.02)$ 分、 $(26.02 \pm 3.99)$ 分, $P>0.05$ 差异无统计学意义。 治疗之后治疗组与对比组患者的平均神经功能缺损评分是

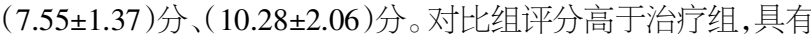
临床不均衡性 $(t=5.0945, P=0.0037)$ 。

\section{4 讨论}

脑卒中一旦发病, 病情发展就非常快, 需要及时就医, 若 是得不到有效治疗, 则会出现较高的致残率与致死率, 给患者 的身心健康带来不良影响。脑卒中抑郁便是较为显著的心理 不良情绪之一, 其体现在心境低落、兴趣下降以及社会适应能 力降低等方面, 这一综合征可发生在脑卒中疾病的各个阶段 中, 同时此综合征的发病机制尚未明确,但是同患者的年龄、 性别以及教育程度等没有关联，主要是同其脑卒中的部位与 神经缺损情况相关联[3,4]。论文中基础治疗具有一定的短期效 果, 但是长期效果不明显; 采用高频重复经颅磁刺激与高压氧 治疗后, 患者治疗后当天, 治疗组中的 HAMD 评分却显著低于 对比组的 HAMD 评分, 组间数据对比有临床比较的意义 $(P>$ $0.05)$ 。治疗之前, 治疗组与对比组的平均神经功能缺损评分是 $(25.17 \pm 4.02)$ 分、 $(26.02 \pm 3.99)$ 分, $P>0.05$ 没意义; 治疗之后治 
医疗卫生设备 Medical Equipments

疗组与对比组患者的平均神经功能缺损评分是 $(7.55 \pm 1.37)$ 分、(10.28 2.06$)$ 分; 对比组评分高于治疗组, 具有临床不均衡 性 $(t=5.0945, P=0.0037)$ 。

得知高频重复经颅磁刺激是利用时变磁场作用于大脑皮 质产生感应电流, 从而使神经细胞的膜电位进行改变, 以引起 去极化，使得脑内代谢和神经电活动产生变化的生物刺激技 术, 患者的局部脑血流量明显增加,进而能够大大改善其抑郁 症状; 同时展开高压氧治疗, 可以帮助患者增加血氧含量, 使 得脑组织的血管供氧得到提高。改善脑部氧代谢及微循环, 有 利于患者脑细胞的修复和神经功能的重建 ${ }^{\left[{ }^{[}\right]}$。

\section{参考文献}

[1]谭伟,余寒,周勇.重复经颅磁刺激对脑卒中后抑有患者影响的 对照研究[J].山西医药杂志,2017,46(8):936-938.
[2]杨柳,刘玉山, 吴宁渤, 等. 低频重复经颖磁刺激对缺血性脑卒中 后抑有槵者血清神经递质、细胞因子的影响[J].海南医学院学报,2017,23 (10):1434-1437.

[3] 韩琦,朱辉,毛梦丹.重复经颖磁刺激对脑卒中后抑郁患者抑有 情绪、睡眠障碍及日常生活活动能力的影响[J].心理医生,2016,22(30): 107-108.

[4]何予工,李鹏.重复经颅磁刺激对脑卒中后抑郁患者抑有情绪、 睡眠障碍及日常生活活动能力的影响 [J].中华物理医学与康复杂志, 2015,37(5):361-364.

[5]刘梅,马金玲,赵艳芳,等.低频重复经颅磁刺激对脑卒中所致抑 郁症的疗效及对血清脑源性神经营养因子和白细胞介素 -6 的影响 [J].中国老年学杂志,2018,38(19):4711-4713. 